International Mathematical Forum, 2, 2007, no. 49, 2437 - 2446

\title{
Foliations and Lichnerowicz Basic Cohomology
}

\author{
Hassan Ait Haddou \\ LAMAV, Universite de Valenciennes, Le Mont Houy-ISTV2 \\ F-59313 Valenciennes Cedex 9, France
}

\begin{abstract}
The aim of this work is to introduce and give some properties of the Lichnerowicz basic cohomology which is an important generalization of the basic cohomology.
\end{abstract}

Mathematics Subject Classification: Primary 47A15; Secondary 46A32, 47D20

Keywords: Lichnerowicz cohomology, basic cohomology, Leray-Hirsch theorem

\section{Introduction}

In this paper, we introduce the Lichnerowicz basic cohomology for a foliated manifold and a basic closed 1-forme $\omega$. Recall that the Lichnerowicz cohomology is the cohomology of the complex of differential forms on a smooth manifold with the de Rham differential operator deformed by a closed 1-form $\omega$. The Lichnerowicz cohomology is a proper tool of locally conformal symplectic geometry, see [11].

In [1] we have showed the Leray-Hirsch theorem for basic and vertical forms. In this note we will generalize this theorem for all differential forms.

In the second section,we first define the Lichnerowicz cohomology and some of its basic properties. In the last section, we introduce the basic Lichnerowicz cohomology for any foliated manifold and show that many properties of the usual basic cohomology still have their analogues within the Lichnerowicz basic cohomology.

\section{The Lichnerowicz cohomology}

Let $\omega$ be a closed 1 -form on $M$. The Lichnerowicz cohomology $H_{d^{\omega}}^{*}(M)$ is the cohomology of the complex $\Omega^{*}(M)$ of differential forms on a smooth manifold 
$M$ with the coboundry operator

$$
d^{\omega}=d+e(\omega)
$$

where $d$ is the exterior derivative and $e(\omega)$ is given by

$$
e(\omega)(\alpha)=\omega \wedge \alpha
$$

for all $\alpha \in \Omega^{*}(M)$.

We will denote by $H_{\omega}^{*}(M)$ the cohomology of the complex $\left(\Omega^{*}(M), d_{\omega}\right)$. Similarly we define the Lichnerowicz-cohomology with compact support $H_{\omega}^{*}(M)_{c}$.

Proposition 2.0.1 ([11]) Let $M$ be a differentiable manifold and $\omega$ a closed 1-form on $M$.

(a) If $\omega$ is exact then $H_{\omega}^{*}(M) \cong H_{d R}^{*}(M)$.

(b) Suppose $\left[\omega^{\prime}\right]=[\omega] \in H^{1}(M)$ : there exists a smooth positive function $f$ such that $\omega^{\prime}=\omega+d(\ln f)$. Then the following application

$$
\begin{aligned}
\varphi: H_{\omega^{\prime}}^{*}(M) & \longrightarrow H_{\omega}^{*}(M) \\
{[\alpha] } & \longmapsto[f \alpha]
\end{aligned}
$$

is an isomorphism.

In this section we recall another interpretation of the Lichnerowicz-cohomology given by Banyaga in [3].

Let $\pi: \tilde{M} \longrightarrow M$ be the minimum regular cover over which the 1 -form $\omega$ pulls back to an exact 1 -form and let $\lambda: \tilde{M} \longrightarrow \mathbb{R}$ be a positive function on $\tilde{M}$ such that $\pi^{*} \omega=d \lambda$. It is well known that the group of automorphisms $\mathcal{A}$ of the covering $\tilde{M}$ is isomorphic to the group of periods of $\omega[12]$.

Lemma 2.0.2 For any $\tau \in \mathcal{A}$, the function $\frac{(\lambda \circ \tau)}{\lambda}$ is independent of the choice of $\lambda$. We denote it by $c_{\tau}$.

The set $\mathcal{F}_{c \mathcal{A}}^{*}(M)$ of all differential forms $\alpha$ on $\tilde{M}$ such that $\tau^{*} \alpha=c_{\tau} \alpha$ for all $\tau \in \mathcal{A}$, is a subcomplex of the de Rham complex of $\tilde{M}$. We denote its cohomology by $H_{c \mathcal{A}}^{*}(M)$. The following theorem is proved in [4]

Theorem 2.0.3 $H_{c \mathcal{A}}^{*}(M)$ is isomorphic with $H_{\omega}^{*}(M)$.

Example 2.1. Let $M=S^{1}$ and $\omega$ be a generator of its first de Rham cohomology. We claim that $H_{\omega}^{0}\left(S^{1}\right)=0$. So let $f \in \Omega^{0}\left(S^{1}\right)$ such that $d f=0$ and $\tau^{*} f=c_{\tau} f$. The condition $d f=0$ means that $f=k$ is a constant. Then, the second condition $k=f_{o \tau}=c_{\tau} k$ translates to $\left(1-c_{\tau}\right) k=0$, but if $\omega$ 
is non-exact, $c_{\tau} \neq 1$, hence $k=0$. So $H_{\omega}^{0}\left(S^{1}\right)=0$ and by Poincaré duality $H_{\omega}^{1}\left(S^{1}\right)=0$.

As in the ordinary the de Rham cohomology case we prove that the cup poduct is an isomorphism, namely we have the following theorem:

\section{Theorem 2.0.4 (Künneth formula) .}

Suppose that $M$ and $N$ have good covers and let $\omega_{1}$ respectively $\omega_{2}$ a closed 1-form on $M$ respectively $N$. Then the map

$$
\cup: H_{\omega_{1}}^{*}(M) \otimes H_{\omega_{2}}^{*}(N) \longrightarrow H_{\omega}^{*}(M \times N)
$$

is an isomorphism.

Example 3.2 Let $G$ be a Lie group with Lie algebra $\mathcal{G}, E \longrightarrow M$ a principal $G$-bundle on $M$, and $\omega$ a connection 1 -form on $E$. Thus $\omega \in \Omega^{1}(E, \mathcal{G})$, and its curvature is given by

$$
\Omega=d \omega+\frac{1}{2}[\omega, \omega] \in \Omega^{2}(E, \mathcal{G}) .
$$

A connection $\omega$ with $\Omega=0$ is a flat connection and a bundle with a flat connection is said to be flat. If $E$ is an $S^{1}$-flat bundle, then $[\omega, \omega]=0$ and therefore $\Omega=d \omega=0$. So $\omega$ is an ordinary closed 1 -form

Suppose that $M$ has a good cover $\mathcal{U}$ and let $U \in \mathcal{U}$. Take $d f$ an exact 1 -form on $U$, then

$$
\begin{aligned}
H_{p r_{2}^{*} d f+\omega_{\mid U \times S^{1}}}^{*}\left(U \times S^{1}\right) & \cong H_{d f}^{*}(U) \otimes H_{\left.\omega\right|_{S}{ }^{1}}^{*}\left(S^{1}\right) \\
& \cong H^{*}(U) \otimes H_{\left.\omega\right|_{S^{1}}}^{*}\left(S^{1}\right) \\
& \cong 0
\end{aligned}
$$

since $H_{\left.\omega\right|_{S^{1}}}^{*}\left(S^{1}\right)=0$.

Similarly, for $V \in \mathcal{U}$, we get

$$
\begin{aligned}
H_{p r_{2}^{*} d f+\omega_{\mid V \times S^{1}}}^{*}\left(V \times S^{1}\right) & \cong H_{d f}^{*}(V) \otimes H_{\left.\omega\right|_{S}{ }^{1}}^{*}\left(S^{1}\right) \\
& \cong H^{*}(V) \otimes H_{\left.\omega\right|_{S^{1}}}^{*}\left(S^{1}\right) \\
& \cong 0
\end{aligned}
$$

and

$$
H_{p r_{2}^{*} d f+\omega_{\mid U \cap V \times S^{1}}^{*}}\left(U \cap V \times S^{1}\right) \cong 0 .
$$

Using the Mayer-Vietoris sequence inductively, we immediately obtain

$$
H_{\omega}^{*}(E) \cong 0
$$


Next we will prove the Leray-Hirsch theorem for the Lichnerowicz cohomology. First we state such theorem for basic and vertical forms and then for general forms.

Theorem 2.0.5 Let $(E, M, F, \pi)$ be a fiber bundle over $M$. Suppose that $M$ has a finite good cover. If there are global cohomology classes $\left(e_{1}, e_{2}, \ldots, e_{r}\right)$ on $E$ which when restricted to each fiber freely generate the cohomology of the fiber, then $H_{\pi^{*} \omega}^{*}(E)$ is a free module over $H_{\omega}^{*}(M)$ with basis $\left(e_{1}, e_{2}, \ldots, e_{r}\right)$, i.e.

$$
H_{\pi^{*} \omega}^{*}(E) \cong H_{\omega}^{*}(M) \otimes \mathbb{R}\left\{e_{1}, \ldots, e_{r}\right\} \cong H_{\omega}^{*}(M) \otimes H^{*}(F) .
$$

By an analogue proof of the previous theorem we can show the following LerayHirsch theorem for vertical forms:

Theorem 2.0.6 Let $(E, M, F, \pi)$ be a fiber bundle over $M$ and $\omega$ a closed 1-form on $E$. Suppose that $M$ has a finite good cover. If there are global cohomology classes $\left(e_{2}, \ldots, e_{r}\right)$ on $E$ such that $\left([\omega]=e_{1}, e_{2}, \ldots, e_{r}\right)$ restricted to each fiber freely generate the cohomology of the fiber, then

$$
H_{\omega}^{*}(E) \cong H^{*}(M) \otimes H_{i^{*} \omega}^{*}(F)
$$

where the map $i: F \longrightarrow E$ is the inclusion.

More generally, we can use the same arguments as before to prove the Leray-Hirsch theorem for any closed form on a manifold, namely we have the following theorem:

Theorem 2.0.7 Let $(E, M, F, \pi)$ be a fiber bundle over $M$ and $\omega$ a closed 1-form on $E$. Suppose that $M$ has a finite good cover. If there are global cohomology classes $\left(e_{2}, \ldots, e_{r}\right)$ on $E$ such that $\left([\omega]=e_{1}, e_{2}, \ldots, e_{r}\right)$ restricted to each fiber freely generate the cohomology of the fiber, then

$$
H_{\omega}^{*}(E) \cong H^{*}(M) \otimes H_{i^{*} \omega}^{*}(F)
$$

where the application $i: F \longrightarrow E$ is the inclusion.

Remark 2.0.8 1. The assumption that $M$ has a finite good cover is necessary for the induction argument.

2. Remark also that the assumption that there are global cohomology classes $\left(e_{1}, e_{2}, \ldots, e_{r}\right)$ on $E$ which when restricted to each fiber freely generate the cohomology of the fiber is necessary because all fibers have the same cohomology but their generators may be different. For example take $x, y$ 
in $M$ and put $E_{x}=\pi^{-1}(x)$ and $E_{y}=\pi^{-1}(y)$. Denote by $\left(u_{1}, u_{2}, \ldots, u_{r}\right)$ resp. $\left(v_{1}, v_{2}, \ldots, v_{r}\right)$ the generators of de Rham cohomology of $E_{x}$ resp. $E_{y}$. We have $H^{*}\left(E_{x}\right) \cong H^{*}\left(E_{y}\right)$, but $\left(u_{i}\right)$ may have no relation with $\left(v_{j}\right)$. The assumption means that the generators of the cohomology of the fibers over intersection are agree since they are restrictions of $e_{i}$.

\section{Lichnerowicz basic cohomology}

Let $(M, \omega)$ be a smooth $n$-dimensional manifold equipped with the closed 1 -form $\omega$ and $\mathcal{F}$ a foliation.

Suppose that $\omega$ is basic, that is $i_{X} \omega=0=L_{X} \omega$. Then $i_{X} d^{\omega} \alpha=0$ and $L_{X} d^{\omega} \alpha=0$,

for all basic $p$-form $\alpha$ on $M$.

Definition 3.0.9 Let $(M, \omega)$ be a smooth manifold together with a basic closed 1-form $\omega$, and $\mathcal{F}$ a foliation on $M$. We denote $H_{b \omega}^{*}(\mathcal{F})$ the cohomology of the complex $\left(\Omega_{b \omega}^{*}(\mathcal{F}), d^{\omega}\right)$ and call it the Lichnerowicz basic cohomology of the foliated manifold $(M, \mathcal{F})$.

Remark 3.0.10 The Lichnerowicz basic cohomology plays the role of the Lichnerowicz cohomology of the leaf space.

Proposition 3.0.11 1. The Lichnerowicz basic cohomology depends only on the basic class of $\omega$. In fact, we have the following isomorphism

$$
H_{b(\omega+d f)}^{*}(\mathcal{F}) \cong H_{b \omega}^{*}(\mathcal{F})
$$

which is given by $\alpha \longmapsto e^{f} \alpha$.

2. Note that $H_{b \omega}^{*}(\mathcal{F})$ is not an algebra but the exterior product induces the following homomorphism

$$
\wedge: H_{b \omega}^{*}(\mathcal{F}) \times H_{b(-\omega)}^{*}(\mathcal{F}) \longrightarrow H_{b}^{*}(\mathcal{F})
$$

Proposition 3.0.12 Let $(M, \mathcal{F})$ a foliated smooth manifold and $\omega$ a closed basic no exact 1 -form on $M$. Then

$$
H_{b \omega}^{0}(\mathcal{F})=\left\{f \in \Omega_{b}^{0}(\mathcal{F}) / d f+f \omega=0\right\}=0 .
$$

\section{Proof:}

Suppose that $d f+f \omega=0$. Then the function $f$ must admit zeros since $\omega$ is not exact. Set $S=\{x \in M / f(x)=0\}$, that is a closed set on $M$.

We prove that it is also open. 
Let $x \in S$ and $U$ an open contractible neighborhood of $x$. Then $\omega_{\left.\right|_{U}}=\frac{d \varphi}{\varphi}$ where $\varphi>0$, and then by the isomorphism

$$
\begin{aligned}
H_{b \omega}^{*}(U) & \longrightarrow H^{0}(U) \\
f & \longmapsto\left[\frac{f}{\varphi}\right]
\end{aligned}
$$

we get that $\frac{f}{\varphi}=$ cte on $U$. Since $\frac{f(x)}{\varphi(x)}=0$ then $f(x)=0$ on $U$. Furthermore, $U \in S$.

If $M$ is connected we get $M=S$, and then $H_{b \omega}^{0}(M)=0$.

Proposition 3.0.13 Let $(M, \mathcal{F})$ be a foliated manifold and $\omega$ a closed 1-form on $M$. The inclusion $\Omega_{B}^{*}(\mathcal{F}) \hookrightarrow \Omega^{*}(M)$ induce an injective map in cohomology $H_{b \omega}^{1}(\mathcal{F}) \hookrightarrow H_{\omega}^{1}(M)$.

\section{Proof:}

Let $\alpha \in \Omega_{b}^{1}(\mathcal{F})$ such that $\alpha=d^{\omega} f$ where $f: M \longrightarrow \mathbb{R}$. Then $\alpha=d f+f \omega$ and $\alpha-f \omega=d f$. Since $\alpha$ and $\omega$ are basic then $f$ is basic and $[\alpha]=0 \in H_{(b \omega)}^{1}(\mathcal{F})$.

Let $M$ be an $n$-dimensional smooth compact manifold, and let $\mathcal{F}$ be a foliation defined by the submersion $\pi: M \longrightarrow W$ of $M$ to a manifold $W$ of dimension $q$.

Suppose that $W$ is connected and oriented, then by Guedira-Lichnerowicz theorem we have

$$
H_{b \omega}^{q}(\mathcal{F})=0
$$

In particular, if $W=S^{1}$ or $T^{q}$, the Lichnerowicz basic cohomology is also trivial.

\section{Gysin Sequence}

The relation between the de Rham cohomology and the basic Lichnerowicz cohomology of $M$ is given by the Gysin sequence. The Gysin sequence for isometric flow is given by

$$
\ldots \longrightarrow H_{b}^{p}(\mathcal{F}) \stackrel{e}{\longrightarrow} H_{b}^{p+2}(\mathcal{F}) \longrightarrow H^{p+2}(M) \longrightarrow H_{b}^{p+1}(\mathcal{F}) \longrightarrow \ldots
$$

where $e$ is the connecting morphism given by multiplication by the Euler class. Recall that the Euler class is the cohomological class of the differential characteristic form $\chi=i_{X} \mu \in \Omega^{1}(M)$, that is,

$$
e=[d \chi] \in H_{b}^{2}(\mathcal{F}) .
$$

This sequence is induced by the short Gysin sequence

$$
0 \longrightarrow \Omega^{*}(M) \longrightarrow \Omega_{b}^{*}(\mathcal{F}) \stackrel{\rho}{\longrightarrow} \frac{\Omega^{*}(M)}{\Omega_{b}^{*}(\mathcal{F})} \longrightarrow 0,
$$

where $\rho$ is the projection induced by the inclusion $\Omega_{b}^{*}(\mathcal{F}) \hookrightarrow \Omega^{*}(M)$. 
Proposition 4.0.14 ([14]) The Euler class e of an isometric flow $\mathcal{F}$ vanishes if and only if there exists a foliation $\mathcal{G}$ transverse to $\mathcal{F}$ which is defined by a cycle.

for a Riemannian flow, one can choose a bundle-like metric $\mu$ such that the mean curvature form $\omega$ is basic and then closed. Thus $[\omega] \in H_{b}^{1}(\mathcal{F})$ is an invariant of the foliation called the Álvarez class.

Let $\mathcal{F}$ a Riemannian flow on a manifold $M$. The Euler form is the 2 -form defined by $d \chi=e+\chi \wedge \omega$.

The Euler form thus defined is basic and satisfies $d^{\omega} e=0$. Then

$$
[e] \in H_{b \omega}^{2}(\mathcal{F}) .
$$

Theorem 4.0.15 ([14]) Let $\mathcal{F}$ be a Riemannian flow on a compact manifold $M$. We have the long exact sequence

$$
\ldots \longrightarrow H_{b \omega}^{p}(\mathcal{F}) \stackrel{e}{\longrightarrow} H_{b}^{p+2}(\mathcal{F}) \longrightarrow H^{p+2}(M) \longrightarrow H_{b k}^{p+1}(\mathcal{F}) \longrightarrow \ldots
$$

Let $M$ be a differentiable 3 -dimensional manifold and $\mathcal{F}$ a Riemannian flow on $M$.

Take $p=1$ in the above Gysin sequence. We get

$$
0 \longrightarrow H_{b \omega}^{0}(\mathcal{F}) \stackrel{e}{\longrightarrow} H_{b}^{2}(\mathcal{F}) \longrightarrow H^{2}(M) \longrightarrow H_{b \omega}^{1}(\mathcal{F}) \longrightarrow H_{b}^{3}(\mathcal{F}) \longrightarrow H^{3}(M)
$$

And then we deduce

$$
H_{b}^{1}(\mathcal{F}) \cong H^{1}(M)
$$

Now, let $M$ be a differentiable $n$-dimensional connect manifold and $\mathcal{F}$ a Riemannian foliation on $M$ with $q=n-1$. Then the Gysin sequence

$$
H_{b}^{n}(\mathcal{F}) \longrightarrow H^{n}(M) \longrightarrow H^{n-1}(\mathcal{F}) \longrightarrow H^{n+1}(\mathcal{F})
$$

becomes

$$
0 \longrightarrow \mathbb{R} \longrightarrow H^{q}(\mathcal{F}) \longrightarrow 0
$$

Then

$$
H^{q}(\mathcal{F}) \cong \mathbb{R}
$$

\section{Invariant of foliation and Lichnerowicz basic cohomology}

We have the following theorem due to Prieto and Saralegi. 
Theorem 5.0.16 ([13]) The Euler $[e] \in H_{b(-\omega)}^{2}(\mathcal{F})$ is an invariant of the foliation and it vanishes if and only if there exists a foliation $\mathcal{G}$ transverse to $\mathcal{F}$ defined by an integrable 1-form $\theta$, such that $d \theta=\tau \wedge \theta$, where $\tau$ is basic.

One can show that the Euler class $[e] \in H_{(b(-\omega)}^{2}(\mathcal{F})$ and the Alvarez class $[\omega] \in H_{b}^{1}(\mathcal{F})$ are independent.

The relation between $[e] \in H_{b(-\omega)}^{2}(\mathcal{F})$ and the Goudbillon-Vey class $G V(\mathcal{G})$ is given by the following proposition.

Proposition 5.0.17 The vanishing of the Euler class of a Riemannien flow is equvalent to the vanishing of Godbillon-Vey invariant of the transverse foliation $\mathcal{G}$.

Proof:

Suppose that $[e] \in H_{b(-\omega)}^{2}(\mathcal{F})=0$. Then there exists $\gamma \in \Omega_{b}^{1}(\mathcal{F})$ such that

$$
\begin{aligned}
d_{-\omega \gamma} & =d \gamma+\omega \wedge \gamma=e \\
& \Longleftrightarrow d \gamma+\omega \wedge \gamma=d \chi+\omega \wedge \chi \\
& \Longleftrightarrow d(\chi-\gamma)=\omega \wedge \gamma-\omega \wedge \chi \\
& \Leftrightarrow d(\chi-\gamma)=\omega \wedge(\chi-\gamma) .
\end{aligned}
$$

We have by (1) that the 1 -forme $\omega=\chi-\gamma$ is integrable, then ker $\omega$ define a foliation $\mathcal{G}$ of codimension 1 .

Remark 5.0.18 The Godbillon-Vey invaraint of foliation $G V(\mathcal{G})=[\omega \wedge d \omega] \in$ $H_{D R}^{3}(M)$ and the Reeb class $\mathcal{R}(\mathcal{G}) \in H^{1}(\mathcal{G})$ of transverse foliation $\mathcal{G}$ are trivial since the 1-form $\omega$ can be taken closed and basic.

The vanishing of the Euler class and the Álvarez class are independent. The following is an example of a Riemannian flow which is not isometric and with a nonzero Euler class.

\section{Example 5.0.19 ([13])}

Let $M^{6}$ be the orbit space of the action

$$
\psi: Z^{2} \times\left(T^{4} \times \mathbb{R}^{2}\right) \longrightarrow\left(T^{4} \times \mathbb{R}^{2}\right)
$$

given by

$$
\psi\left((k, l),\left[y_{1}, y_{2}, z_{1}, z_{2}\right],(t, x)\right)=\left(B^{k} \circ I^{l}\left(\left[y_{1}, y_{2}, z_{1}, z_{2}\right]\right),(x+k, t+l)\right)
$$


where $B, I \in S L(4, Z)$ defined y

$$
B=\left(\begin{array}{cc}
A & 0 \\
0 & A
\end{array}\right) \text { et } I=\left(\begin{array}{cc}
i d_{2} & 0 \\
0 & I d_{2}
\end{array}\right)
$$

and

$$
A=\left(\begin{array}{ll}
2 & 1 \\
1 & 1
\end{array}\right)
$$

It is the fibration $\pi: M^{6} \longrightarrow T^{2}$ with fiber $T^{4}$. Take a metric $\mu$ on $M^{6}$ such that the parallelism $\left\{X, T, Y_{1}, Y_{2}, Z_{1}, Z_{2}\right\}$ is orthonormal and let $\{\alpha, \beta, \gamma, \eta\}$ the dual basis of 1 -forms. The flow $\mathcal{F}$ defined by $Y_{1}$ is Riemannian with respect to the metric $\mu$ such that $L_{Y_{1}} \mu_{Q}=0$.

One have

$$
\chi=\gamma_{1}, \quad \omega=(\log \lambda) \alpha, \quad e=-\beta \wedge \eta .
$$

Let $\theta$ be the generator of $H^{1}\left(M^{6}\right)$. Since $[\alpha]=\pi^{*}([\theta])$, the Álvarez class of the $\mathcal{F}$ is nonzero.

We can show there exist no basic 1-form theta such that $d_{-\omega} \theta=e$.

This is a new example of non trivial Lichnerowicz cohomology group.

ACKNOWLEDGEMENTS. This work was prepared during my PostDoc visit at The University of Valenciennes. I would like to express deep gratitude to Aziz El Kacimi for his warm hospitality and his permanent encouragement. His guidance and support were crucial for the successful completion of this project.

\section{References}

[1] H. Ait Haddou, On the Leray-Hirsch theorem for Lichnerowicz cohomology, Afrika Mat. (3) 16 (2005), 33-42.

[2] L., Auslander, L. Green and F., Hahn, Flows on Homogeneous spaces, Annals of Maths. Studies 53, Princeton Univ. Press, 1963.

[3] A. Banyaga, Some properties of locally conformal symplectic structures, Comment Math.Helv. 77 (2002) 383-398. 49.

[4] A. Banyaga, A geometric integration of the extended Lee homomorphism, Journal of Geometry and Physics, 39(2001) 30-40.

[5] A. Banyaga, Quelques invariants des structures localement conformément symplectiques,C.R.Acad. Sci. Paris, Serie 1 (2001) 29-32. 
[6] A. Banyaga, On the geometry of locally conformal symplectic manifolds, Infinite dimentional Lie groups In Geometry and representation theory, A. Banyaga, J.Leslie and T. Robart Ed. World Scientific 2002 pp 79-91.

[7] A. Banyaga, Examples of non $d_{\omega}$-exact locally conformal symplectic forms, arXiv:math/0308167.

[8] R. Bott and L. W. Tu, Differential Forms in Algebraic Topology, GTM 82, Springer-Verlag, New York Heidelberg Berlin,(1982).

[9] C.Godbillon, Eléments de topologie algèbrique, Hermann, Paris, (1971), 45-76.

[10] F. Guerida and A. Lichnerowicz, Géométrie des algèbres de Lie locales de Kirilov, J.Math. Pures et Appl. 63(1984), 407-484.

[11] S. Haller and T. Rybicki, On the group of diffeomorphisms preserving a locally conformal symplectic structure. Ann. Global Anal. and Geom. 17(1999), 475-502.

[12] C. Godbillon and J. Vey, Un invariant des feuilletages de codimension 1, C.R. Acad Sci. Paris, Serie A 273 (1971),92-95.

[13] J. I. Prieto et M. Saralegi, Cohomology of Riemannian flows, Proceeding of the IX Workshop i la Geltru, 2000.

[14] M. Saralegi, The Euler class for isometric flows, Pitman Research Notes in Math. 131 (1985) 220-227.

Received: January 31, 2007 